\title{
METODOLOGÍA \\ COMUNICATIVA EN EDUCACIÓN DE PERSONAS ADULTAS
}

\author{
Ramón Flecha* \\ Itxaso Tellado**
}

\begin{abstract}
RESÚMEN: Este artículo analiza la metodología de investigación comunicativa para la transformación social de la educación de personas adultas. A partir de sus elementos clave: inclusión de las voces de las personas participantes, ruptura del desnivel metodológico y reconocimiento de la inteligencia cultural. Esta metodología ha sido la base con la que investigaciones del más alto rango científico europeo han logrado un destacado impacto científico, político y social al generar actuaciones de educación de personas adultas con las cuales los colectivos vulnerables superan la exclusión social y promueven su prevención.
\end{abstract}

Palavras-clave: Metodología comunicativa. Educación de personas adultas. Impacto. Colectivos vulnerables.

\footnotetext{
* Universidad de Barcelona, Departamento de Teoría Sociológica, Filosofía del Derecho y Metodología de las Ciencias Sociales, Barcelona, Espańa. E-mail de contacto: ramon. flecha@ub.edu.

** Universidad de Vic-Universidad Central de Cataluña, Departamento de Pedagogía. Vic, Espańa. E-mail de contacto: itxaso.tellado@uvic.cat.
} 


\title{
Communicative methodology in adult education
}

\begin{abstract}
This article analyzes the communicative research methodology for the social transformation of adult education, based on its key elements: the inclusion of the participants' voices, the rupture of the methodological gap and the acknowledgement of cultural intelligence. This methodology has been the basis with which research at the highest European scientific rank has achieved scientific, political and social impact when developing adult educational actions through which vulnerable groups have overcome social exclusion and promoted its prevention.
\end{abstract}

Keywords: Communicative methodology. Adult education. Impact. Vulnerable groups.

La metodología de investigación comunicativa es la respuesta metodológica al giro dialógico de las sociedades $y$ las ciencias que ya han tenido un impacto importante en la transformación de las situaciones de desigualdad y exclusión. La investigación llevada a cabo con la metodología comunicativa implica un diálogo continuo e igualitario entre investigadores y las personas de la comunidad o realidad estudiada. (GÓMEZ; PUIGVERT; FLECHA, 2011, p. 235)

urora es una mujer de mediana edad. Está entre el público de un acto académico, que se ha organizado en la Universidad 1 de Barcelona, con motivo de la visita de profesor Jürgen Habermas, reconocido filósofo de las ciencias sociales, a nivel internacional. Aurora es una mujer que se ha alfabetizado de adulta. Es un ejemplo de tenacidad y de voluntad para superar las dificultades que ha vivido a lo largo de su vida. Levanta la mano y realiza la pregunta. Un rumor de murmullos surge de entre las filas de asistentes, atestadas de profesores y catedráticos. ¿Cómo puede ser que una persona no académica pregunte al mejor científico social del momento? El profesor Habermas escucha la pregunta atentamente. Dice: ha hecho usted una pregunta brillante y crítica. A continuación pasa a responderle. 
El diálogo entre Aurora y Jürgen Habermas es un ejemplo que ilustra perfectamente la ruptura del desnivel metodológico que presentan Gómez, Latorre, Sánchez y Flecha (2006) en su libro Metodología Comunicativa Critica. Todas las personas disponemos de "inteligencia cultural". La investigación en ciencias sociales (COLE; SCRIBNER, 1974; ROGOFF, 1990, 2003; MOLL 1992, 2004) ha demostrado ya que la inteligencia es un rasgo humano que aparece en todas las culturas, en todos los lugares del mundo, en cualquier momento histórico. No hay personas sin inteligencia. Por tanto, todo el mundo podemos aportar nuestros respectivos saberes y experiencias a la creación de conocimiento relevante. Habermas en 2001 era muy consciente del increíble potencial del diálogo igualitario para llegar a consensos que amplían definitivamente los límites de nuestro acervo de conocimiento. Él, como otros muchos autores internacionalmente reconocidos por sus aportaciones, como Paulo Freire, siempre ha reivindicado la necesidad de incluir todas las voces en el diálogo que emerge del conocimiento. La metodología comunicativa tiene ese espíritu transformador y profundamente crítico, porque parte de la premisa del diálogo igualitario (FLECHA, 2000) que se basa en defender los argumentos con pretensiones de validez. Esta capacidad para incluir las voces desde la premisa de la generación de conocimiento científico compartido a través del diálogo igualitario, es una de las razones por las que organismos internacionales, como la Comisión Europea han reconocido a la metodología comunicativa como un enfoque particularmente apropiado para trabajar con personas de grupos vulnerables para lograr un impacto social de transformación que lleve a superar las situaciones de exclusión en las que viven. (PARLAMENTO EUROPEO, 2005)

En este artículo se exponen las bases científicas de la metodología comunicativa, y se examina el impacto que tiene dicha metodología en la validez de la investigación que realizamos en las ciencias sociales. La inclusión de las voces de personas pertenecientes a grupos vulnerables que nunca acostumbran a tener la oportunidad de hacer valer sus conocimientos en el ámbito académico, amplía y mejora de manera innegable las aportaciones científicas que podemos hacer con nuestro trabajo. Esto abre la puerta hacia una transformación social real, partiendo de una investigación responsable, rigurosa y comprometida. 


\section{EL DIÁLOGO IGUALITARIO}

En 1931 García Lorca creó junto con cuatro estudiantes de filosofía y letras, y cuatro más de arquitectura, el teatro de La Barraca, que llevó los clásicos de la literatura universal a pueblos de todo el Estado Español. Lorca fue el más grande dramaturgo y poeta, del siglo XX, de las letras hispánicas. Su genialidad fue poner al nivel de los clásicos las vidas cotidianas de personas corrientes, demostrando así cuánto saber hay en sus hechos más nimios. Habermas en 1984 publicó un libro que nos ha dado mucho que pensar a las personas que nos dedicamos a la investigación en ciencias sociales. No fue el primero en plantear la posibilidad de tener en cuenta las voces de personas no académicas; pero sí que el rango indiscutible de su aportación puso sobre la palestra un tema tan crucial en nuestro trabajo. Habermas, en el primer volumen de $\mathrm{La}$ Teoría de la Acción Comunicativa, ese libro que publicó en 1984, realiza un análisis exhaustivo de la teoría de la argumentación, desde el punto de vista de la racionalidad. A lo largo de las páginas, se da cuenta que en todo proceso argumentativo existen dos fuentes de legitimidad, muy diferentes entre sí: las argumentaciones sobre pretensiones de poder, y las argumentaciones sobre pretensiones de validez. Habermas plantea que las ciencias sociales tienen que moverse desde la fenomenología hacia la intersubjetividad, porque según él la realidad social se construye desde "dentro", como resultado de los actos de habla de personas que son sujetos capaces de lenguaje y acción. El diálogo que se propone buscar es el entendimiento y partiendo de la racionalidad comunicativa. (HABERMAS, 1987a; 1987b) Es decir, que las personas estudiadas en una investigación puedan saber detalles de la investigación, que aporten información pero también interpretación o incluso redacción de propuestas, orientaciones y conclusiones.

El diálogo igualitario que se plantea en Compartiendo Palabras nace del reconocimiento de ese valor universal señalado por Habermas (la gramática generativa universal de la que habla Chomsky), según el cual todas las personas somos sujetos capaces de lenguaje y acción. En la medida que esto es cierto, significa que todas las personas podemos aportar argumentos susceptibles de cumplir con las reglas de la inteligibilidad, a saber: que los argumentos tienen que ser (1) válidos en la medida 
que reflejan una experiencia o un hecho; (2) veraces desde el punto de vista de las intenciones del hablante; y (3) normativamente correctos. Cuando dos o más personas participan en un diálogo igualitario, lo que las mueve en caso de estarse produciendo una interacción de tipo comunicativo es que quieren llegar a consensos sobre el objeto discutido. En la investigación este afán por alcanzar acuerdos y consensos se traduce en la creación compartida de conocimiento en base a evidencias que se asientan sobre las ideas de validez, veracidad y rectitud.

La metodología comunicativa promueve y toma como herramienta central el diálogo y la participación en la construcción del conocimiento, en tanto que no prioriza la acumulación de información sino su uso y sus resultados. (TELLADO, 2007) A través de la creación de espacios de diálogo igualitario, la metodología comunicativa crea las condiciones para que este tipo de conocimiento compartido emerja. Jesús Gómez en 2006 publicó junto con sus compañeros/as el libro $\mathrm{Me-}$ todología Comunicativa Critica donde recoge los siete postulados de este marco metodológico, que incluyen: la universalidad del lenguaje y de la acción, la asunción de las personas como agentes sociales transformadores, la racionalidad comunicativa, el sentido común, la desaparición del presupuesto de jerarquía interpretativa, el igual nivel epistemológico, y el conocimiento dialógico. En el marco del proyecto RTD Workaló, organizado y dirigido por CREA, en uno de los seminarios para la presentación de resultados al que se había invitado personas del colectivo investigado en este caso comunidad gitana, una mujer gitana joven levantó la mano al terminar la intervención del mayor experto en temas interculturales en Europa, y cuestionó el nuevo concepto de mestizaje que ese experto, Michel Wiewiorka, estaba explicando. Con este ejemplo queremos mostrar precisamente la potencia que tiene este enfoque metodológico en la creación de conocimiento real y con impacto social sobre el mundo de la vida cotidiana de [todas] las personas. La mujer gitana joven argumentaba que ella se sentía igualmente gitana y ciudadana francesa, y que no podía definir su identidad como una suma de identidades parciales, sino como un todo global. La respuesta de Wiewiorka dejó claro que la aportación de esta mujer le había permitido mejorar de manera muy significativa su concepto. 


\section{LA INCLUSIÓN DE TODAS LAS VOCES Y EL IMPACTO SOBRE LA VALIDEZ DE LA INVESTIGACIÓN}

Entre 2001 y 2005 llevamos a cabo la investigación AMAL Inmigración y mercado laboral, financiada por el Plan Nacional de I+D+i del Ministerio de Ciencia y Tecnología, del Estado Español. Fue un estudio complicado, por el tema y el momento histórico. El objetivo era definir estrategias sociales y formativas para superar la exclusión laboral de los inmigrantes, en concreto de las personas procedentes de países árabes y/o musulmanes. El 11-S marcó un hito que afectó de manera crucial al desarrollo de la investigación. En el equipo de trabajo había una persona marroquí y otra originaria de Senegal. Fueron esas dos personas las que permitieron completar el trabajo de campo con una comunidad que se veía criminalizada debido al atentado de las Torres Gemelas. La validez de las conclusiones que se obtuvieron en aquel estudio pasa por la veracidad de las informaciones recogidas durante el trabajo de campo. Gracias al enfoque inclusivo de la metodología comunicativa fuimos capaces de superar las barreras no solo culturales, sino también coyunturales de la situación tan difícil que se había creado.

Uno de los postulados clave de la metodología comunicativa es el igual nivel epistemológico. De acuerdo con este postulado, entendemos que la metodología comunicativa rompe con el desnivel metodológicamente relevante entre las personas que investigan y las que son "investigadas". Tal y como afirma Gómez et al (2006), si queremos comprender un fenómeno, como es el caso de la inmigración árabe y/o musulmana en la Península Ibérica, las personas que investigan tienen que participar en el proceso comunicativo de creación de conocimiento en el mismo plano de igualdad que aquellas personas que son investigadas. En ese sentido, desde este enfoque metodológico, uno de los instrumentos más relevantes es la creación de lo que se denomina como consejos asesores (CA). Los CA están formados por personas pertenecientes a los colectivos que participan en la investigación (los grupos vulnerables, víctimas de la situación de exclusión que está siendo investigada). Estos consejos funcionan en base al criterio de diálogo igualitario, explicado más arriba. Las personas que participan aportan sus argumentos con pretensiones de validez, para contrastar en un diálogo compartido las afirmaciones que 
se realizan en los informes de análisis del trabajo de campo que realizan las personas investigadoras. Cada participante aporta su respectivo conocimiento, de manera que el diálogo se enriquece de las aportaciones de todas las personas, académicas y no académicas.

En otro proyecto, esta vez sobre el concepto de ciudadanía, Citiroma, el CA redefinió qué significa "ciudadanía" en el contexto de las sociedades dialógicas actuales, donde la convivencia de personas de culturas diferentes había transformado "la calle" en unos pocos años.

Nosotros la ciudadanía la entendemos como que en este momento hay como dos aspectos, uno es que estamos conviviendo personas... no es que ahora sea, si no que ahora hay como un auge y se hace visible, siempre ha sido asi y vosotros más que nadie lo habéis vivido. Pero ahora hay un auge como de vivir entre muchas personas, de origenes $y$ de culturas, diferentes. Entonces te vayas al barrio que te vayas, estés en el sitio que estés pues te encuentras con esto, con gente con trayectorias culturales muy diferentes. (CONSEJO ASESOR DE CITIROMA, 2008)

La metodología comunicativa también se basa en el principio de inteligencia cultural (RACIONERO; VALLS, 2008) que indica que cada individuo tiene habilidades comunicativas, prácticas y académicas. Ramis y Krastina (2010) apuntan que "[...] la inteligencia cultural consiste en la inteligencia académica (adquirida en contextos académicos), inteligencia práctica (adquirida en contextos diarios) y la inteligencia comunicativa [...]” (p.244). Esta idea supera la exclusión que sufren muchos grupos de la sociedad de los que se considera que no tienen las habilidades necesarias para participar en actividades académicas. De hecho, mediante la metodología comunicativa se constata que las personas de minorías culturales, personas que viven en barrios pobres y marginalizados, y las personas que tienen poca formación académica pueden hacer contribuciones significativas a la investigación, desde el diseño hasta el análisis y difusión de los principales resultados. 


\section{LA TRANSFORMACIÓN SOCIAL DESDE LA INVESTIGACIÓN COMPARTIDA}

El proyecto europeo INCLUD-ED, Strategies for Inclusion and Social Cohesion in Europe from Education (CREA, 2006-2011), es la investigación de mayor rango científico y con la mayor dotación de recursos dedicados para estudiar la educación escolar en el marco del 6o Programa Marco Europeo de investigación e innovación, y utiliza la metodología comunicativa de investigación. En el marco de esta investigación se estudiaron proyectos locales para la cohesión social analizando comunidades involucradas en proyectos educativos que promuevan la interacción social e intervenciones educativas para contribuir a la reducción de las desigualdades y la marginalización. El único proyecto sobre educación de personas adultas seleccionado para estudiar y poder contribuir así a reducir desigualdades o marginalización fue el de la escuela de personas adultas La Verneda-Sant Martí y su vecindario. Se identificaron actuaciones de éxito en diversas áreas sociales. Estas transformaciones dialógicas afectan muchas áreas de la sociedad, como la política, la económica, la salud, la social, cultural y esferas personales. Las formas de participación en la investigación son múltiples, mediante la participación directa de las personas que están aprendiendo en la escuela de personas adultas en los consejos asesores, o como un miembro más del equipo que diseńa y prepara el proyecto, entre otros. La propia organización comunicativa de los proyectos de investigación permite desarrollar conocimiento nuevo, poniendo en comunicación los conocimientos del colectivo afectado y los conocimientos científicos de las personas investigadoras. Tellado, Serrano y Portell (2013) apuntan que la participación en proyectos de investigación mediante una metodología comunicativa, de las personas que asisten a la escuela de personas adultas La Verneda-Sant Martí, ha tenido un impacto social y político además de científico ya que en el barrio cuenta con mejoras substanciales que se han llevado a cabo en espacios urbanos, de infraestructuras, de vivienda, de transporte y de comunicaciones, pero también en la vida asociativa de este barrio.

Son muchas las experiencias de investigación mediante la metodología comunicativa en las que la participación directa de las personas participantes en la investigación hace replantear las orientaciones de la 
misma. De nuevo tomando el ejemplo de los consejos asesores, son unos espacios en los que surgen temas y cuestiones en el que las personas que participan expresan sus necesidades, en este caso sobre la convivencia y la superación de las discriminaciones hacia la comunidad gitana, además de reclamar un reconocimiento social y cultural. Un hombre gitano expresaba sobre la ciudadanía:

De alguna manera ahora hay una explosión de todo esto y se hace mucho más visible. Entonces, en la ciudadanía por un lado está conviviendo gente diferente pero por otro lado no hay un marco legal que nos identifique a todos, no hay leyes que digan pues siendo diferentes vamos a respetar pues lo que le pasaba a la nena en Madrid y el juicio todavía dura de si le tienen que dar la viudedad o no le tienen que dar la viudedad. ¿Por qué? Pues porque se casó por el rito gitano, se quedó viuda y en la cartilla constaba pero ella no tenía ningún papel de que estaba casada con su marido. Entonces, ¿por qué? Por qué no se puede aceptar la trayectoria cultural de cada uno, por qué no hay un marco legal. Pero claro para todo esto, para que se de este marco legal hay que hacer el reconocimiento, si no se hace el reconocimiento nada. (CONSEJO ASESOR DE CITIROMA, 2008)

Este participante de la investigación contribuye a la investigación con su conocimiento de la realidad al mismo tiempo que reclama para todos (equipo investigador y sociedad civil) y para sí mismo que esta necesidad traspase el espacio de la investigación y llegue a todas las personas.

\section{PALABRAS FINALES}

Los resultados de la aplicación de la metodología comunicativa permiten a las investigaciones partir de la realidad de las personas investigadas y aporta elementos clave para la inclusión o la exclusión social de los colectivos más vulnerables, además del impacto social y 
político que se desarrolla en el propio proceso de la investigación llevada a cabo mediante esta metodología.

Díez-Palomar, Pitanga y Cifuentes (2013) apuntan que "[...] autores como Freire (1998), Kincheloe (2001) y Flecha (2000) han creado un marco teórico que une el rigor científico de investigación con la habilidad de transformar la realidad social [...]” (p. 206). También indican que la diferencia con otras metodologías cualitativas en las que se incorpora el punto de vista del sujeto como son la investigación acción (KEMMIS; MCTAGGART, 2005), indagación dialógica (WELLS, 1999) o enfoques feministas (BUTLER, 1993/2011), entre otros, es que la metodología comunicativa parte de la igualdad epistemológica y ontológica de todos los agentes y actores participantes en el proceso de investigación. Mediante este dialogo la inteligencia cultural de todos los sujetos se puede incorporar.

En la investigación de educación de personas adultas mediante la inclusión de las voces de las personas participantes, la ruptura del desnivel metodológico y el reconocimiento de la inteligencia cultural de la metodología comunicativa, se logra llevar a cabo investigaciones con impacto científico, político y social superando la exclusión social y promoviendo su prevención.

\section{REFERÊNCIAS}

BUTLER, J. Bodies that matter. New York: Routledge. 1993/2011.

CITIROMA. Análisis de nuevos mecanismos de construcción de la ciudadanía multicultural en España: contribuciones del pueblo gitano. Programa Nacional del Plan Nacional de Investigación Científica, Desarrollo e Innovación Tecnológica 2004-2007 I+D MEC. 2006.

COLE, M.; SCRIBNER, S. Culture and thought: a psychological introduction. New York: Wiley, 1974.

CREA. AMAL: Inmigración y mercado laboral. Plan Nacional I+D+I. Programa de socioeconomía. Ministerio de Ciencia y Tecnología. Secretaría de Estado de Política Científica y Tecnología. Investigador principal: Prof. Ramón Flecha, 2001-2005. 
CREA. The creation of new occupational patterns for cultural minorities: the gypsy case-Workaló. Brussels: European Commission, 2004.

. INCLUD-ED Project. Strategies for inclusion and social cohesion in Europe from education. 6th Framework Programme. Citizens and Governance in a Knowledge-based Society. CIT4-CT-2006-028603. Directorate-General for Research, European Commission, 2006-2011.

DÍEZ-PALOMAR, J.; PITANGA, T.; CIFUENTES, P. La Paz School. From a ghetto to a magnet school. International Review of Qualitative Research, v. 6, n. 2, 2013, p. 198-209. [Doi: 10.1525/irqr.2013.6.2.198].

GÓMEZ, J. et al. Metodología comunicativa crítica. Barcelona: El Roure, 2006.

GÓMEZ, A.; PUIGVERT, L.; FLECHA, R. Critical communicative methodology: Informing real social transformation through research. Qualitative Inquiry, n. 17, 2011, p. 235-245. [Doi: 10.1177/1077800410397802].

FLECHA, R. Sharing words. Lanham: Rowman \& Littlefield, 2000.

HABERMAS, J. Teoría de la acción comunicativa. v. I. Racionalidad de la acción y racionalización social. Madrid: Taurus, 1987a.

. Teoría de la acción comunicativa. v. II. Crítica de la razón funcionalista. Madrid: Taurus, 1987b.

KEMMIS, S.; MCTAGGART, R. Participatory action research: communicative action and the public sphere. In: DENZIN, N. K.; LINCOLN, Y.S. (Eds.). The Sage handbook of qualitative research. 3. ed., Thousand Oaks, CA: Sage, 2005, p. 559-603.

MOLL, L. et al. Funds of knowledge for teaching: Using a qualitative approach to connect homes and classrooms. Theory into Practice, v. 31, n. 2, p. 132-141, 1992.

MOLL, L.; GONZÁLEZ, N. Engaging life: A funds of knowledge approach to multicultural education. In: BANKS, J.; BANKS, M. C. (Eds.). Handbook of research on multicultural education. New York: Macmillan, 2004, p. 628-634.

PARLAMENTO EUROPEO. European Parliament resolution on the situation of Roma people in the European Union. 2005. Disponível em <http://www. europarl.europa.eu/sides/getDoc.do?type=MOTION\&reference=B6-20050274\&language $=\mathrm{EN}>$.

RACIONERO, S.; VALLS, R. Communicative approach to teaching and learning. En: KINCHELOE, J. L.; HORN, R. A. (Eds.). The praeger handbook 
of education and psychology, v. 3. Westport, London: Greenwood Publishing Group, 2008, p. 548-557.

RAMIS, M.; KRASTINA, L. Cultural Intelligence in the School. Revista de Psicodidáctica, v. 15, n. 2, 2010, p. 239-252.

ROGOFF, B. Apprenticeship in thinking: cognitive development in social context. New York: Oxford University Press, 1990.

. The cultural nature of human development. New York: Oxford University Press, 2003.

TELLADO, I. Análisis de las percepciones de las personas participantes en la gestión de la escuela de adultos. In: INTERNATIONAL CONGRESS OF QUALITATIVE INQUIRY. 3. 2007. University of Illinois at Urbana-Champaign, p. 395, 2007.

TELLADO, I; SERRANO, M.; PORTELL, D. The achieved Dreams of a neighborhood. International Review of Qualitative Research, v. 6, n. 2, 2013, p. 289-306.

WELLS, G. Dialogic inquiry: towards a sociocultural practice and theory of education. Cambridge: Cambridge University Press, 1999.

Recebido em 20 de fevereiro de 2015.

Aprovado em 29 de maio de 2015.

DOI: http://dx.doi.org/10.1590/CC0101-32622015723765 